\title{
Ethical Issues Raised for Public Health and Socio- Behavioral Research Focused on Containment of the COVID-19 Pandemic
}

\author{
Nyein Su Aye ${ }^{1}$, Khin Hnin Pwint ${ }^{2}$, Hsu Mon Aung 3 , Phyo Yadanar \\ Kyaw Lwin Show ${ }^{5}$, Theingi Thwin ${ }^{6}$, Khin Thet Wai ${ }^{7}$ \\ ${ }^{1}$ Member, Institutional Review Board (IRB), Department of Medical Research. (DMR), \\ ${ }^{2}$ Member, Institutional Review Board (IRB), Department of Medical Research. (DMR), \\ ${ }^{3}$ Secrteriat Member, Institutional Review Board (IRB), Department of Medical Research. \\ (DMR), \\ ${ }^{4}$ Secrteriat Member, Institutional Review Board (IRB), Department of Medical Research. \\ (DMR), \\ ${ }^{5}$ Doctoral Student, Khonkaen University, Thailand, \\ ${ }^{6}$ Director (Retired), Member, IRB (DMR), \\ ${ }^{7}$ Director (Retired), DMR, Independent Consultant \\ 1,2,3,4,6,7No. 5, Ziwaka Road, Dagon Township, Yangon 11191, Myanmar \\ Corresponding Author: Khin Thet Wai \\ E-mail: khinthetwaidmr@gmail.com
}

\begin{abstract}
In response to a global public health emergency, it is critical to focus ethical considerations in public health and socio-behavioral research proposals concerning COVID-19 containment. A desk-based review during October 2020 analyzed two mixed methods studies and three quantitative observational studies submitted to the Institutional Review Board (IRB) of which one study emphasized the Case Report Forms (CRFs) and the rest were online surveys. Main tools intended for primary data collection in the submitted research proposals covered the google forms, Facebook messenger, and mobile phones to access the general public, healthcare providers, and civil society organizations. The waiver of the documented 'Informed Consent' to decide for filling the online google forms, intensive pretesting and data collector training for interviews via digital platforms, and data security issues were priority ethical concerns addressed during the IRB appraisal in addition to preserve anonymity in retrieving and reporting the findings in CRF. The virtual dissemination plan is acceptable during the pandemic. A regional networking scheme of IRBs could foster ways to deal with ethical dilemmas during the public health emergency especially when using the internet and other digital platforms for data collection.

Keywords: Ethical concerns, COVID-19 pandemic, IRB, public health, Socio-behavioural research, Containment
\end{abstract}

\section{INTRODUCTION}

In the upsurge of COVID-19 infection which is unprecedented as a global public health emergency, the research questions in public health and socio-behavioral dimensions are essential for the containment in a real-time support of evidence-informed decisions [1-3]. In light of uncertainties in vaccines and therapeutic agents as well as expanding community transmission of COVID-19 
infection, researchers focus to generate data on non-pharmaceutical interventions to follow the example of earlier pandemics [4]. The local Institutional Review Boards (IRB) might face some difficulties in reviewing those researches during public health emergency in response to pressure from the authorities concerned [5-8]. In the context of COVID 19 pandemic, WHO has introduced the standard operating procedures for rapid reviews by research ethics committees [9-10].

As stated in the Belmont report, the rights of research participants related to safety and beneficence must be considered first [11]. Research has to follow the ethical principles even in the crisis of public health aspect. The explicit ethical concerns for public health and socio-behavioral research depend upon the choice of study design, recruitment approaches and data collection portals, data curation, data sharing and dissemination. Scientific justification, assessment of risks and potential benefits, consultation and engagement, coordination, participant selection, expert review and informed consent were the key factors [12]. The underlying reasons for limited exploration of ethical concerns in IRBs for socio-behavioral research in COVID-19 included lack of familiarity and limited capacity of IRB members and potential solutions exist such as efforts in capacity building [13]. Given that the IRB should consider whether the proposed informed consent taking process is most appropriate to conduct in the situation, it is crucial to seek for any other requirements being justifiable to express the respect of participants [14]. An IRB could waive the informed consent and modify the elements such as the requirement of signature of participants in the context of minimal risk study, if waiving of the informed consent could not severely affect the participant's welfare [15].

Myanmar has reported the first two confirmed cases of COVID-19 on 23 March 2020. Thereafter in April, the accelerated health sector responses covered the guidelines for the healthcare providers for prevention and containment measures. Other strategic approaches covered the establishment of the national volunteer steering unit, community fever clinics in Yangon and Mandalay cities and the National Call Center at the Department of Medical Research [16]. In response to the fulfilment of knowledge gaps for the strengthening of policy and procedures, the research proposals target the perspectives of healthcare providers towards compliance to guidelines, and public understanding of rapidly evolving COVID-19 related information mainly essential for changes in preventive behaviors and to promote early help-seeking for suspected symptoms. The IRB of Department of Medical Research (DMR), Myanmar has received a total of 108 applications for the full-board meetings, expedited reviews and exemption between April to October 2020 during the pandemic period. The new SOP introduced for expedient reviews assisted the IRB members to prepare for quality reviews. Above all, socio-behavioral and public health research contributed for $45 \%(49 / 108)$ of which $24 \%(12 / 49)$ were COVID-19 related proposals. The turnaround time from the date of review to approval by the IRB was as quick as six days in terms of the expedited review process of the COVID-19 related proposals [17].

As such, the IRBs face ethical challenges in balancing the community and individual risks and benefits in newly developed proposals submitted for review during the pandemic period with an emphasis on containment measures. It is imperative to seek for specific ethical considerations to protect human research participants during public health and socio-behavioral research in time of public health emergency response. Both researchers and IRBs are responsible for ensuring the justifiable risk-benefit ratio, social justice, and social values [15]. Studies are limited or none in Myanmar and elsewhere according to the PUBMED literature search in addressing the problem concerning ethical considerations of public health and socio-behavioral research during the COVID-19 pandemic. Moreover, there is a need to fulfil the knowledge gap in research ethics reviews. Therefore, this current study aimed to analyse the ethical concerns in reviewing the COVID-19 related public health and socio-behavioral research proposals submitted to the IRB (DMR), Myanmar between April to October 2020.

\section{METHODOLOGY}

\section{Study design and study population}

A cross-sectional study that included a desk-based record review of IRB at the Department of Medical Research (DMR), Myanmar was carried out in October 2020. Five out of 12 records of 
public health and socio-behavioral research related to COVID-19 containment measures were retrieved from the IRB (DMR).

\section{Sampling and data analysis}

The records at the IRB were purposively selected for the brief review and analys ofed the key features in form of case studies that encompassed the proposal summary and ethical considerations. The contents of the proposal review form by the standard operating procedures of the IRB (DMR) covered: both technical and ethical issues to strengthen scientific integrity.

\section{RESULTS}

Two studies were intended for mixed methods and the remaining three were quantitative observational studies of which one study focused on Case Report Forms (CRFs) of hospitalized COVID-19 patients for secondary data collection and the rest planned online surveys for primary data collection. All were stratified as the minimal risk and were eligible for the expedited review at the IRB. The researchers aimed to use google forms, Facebook messenger, and mobile phones to access the general public, healthcare providers and civil society organizations

Case study 1 emphasized the preparedness and response of health care providers in both public and private sectors related to COVID-19 outbreak in country X (Text box 1).

\section{Text box (1)}

Case study on COVID-19 preparedness and response among public and private health care providers

Project summary

A cross-sectional quantitative online survey is planned for rapid assessment of the preparedness and response to COVID-19. The target population will be 30,000 public and 5,000 private health care providers in country X. The proposed recruitment is by the announcement in the Facebook page along with the provision of a valid URL to download Google Forms in a local language to collect the individual responses. This study will identify the types and sources of information for COVID-19, common and preferred channels among health care providers, and with implementing partner organizations for disease response, awareness on prevention of COVID-19 focusing on physical distancing, about quarantine, signs, symptoms, severity of the disease, reporting and referral procedures of suspected cases and personal preventive measures, perceived needs and the availability of essential equipment support for COVID-19 response and the perceived challenges during COVID-19 response, and their suggestions for improvement.

In case study 1 , the IRB has identified the ethical concerns to:

- Add the recruitment procedure in brief in the form of an announcement/advertisement in Facebook to participate in the online survey before description of the study tool (online google form) in Methodology section;

- Include the announcement in Facebook concerning the general information of research, eligibility to participate, valid survey period, strict confidentiality measures to be undertaken and a valid URL that linked to the survey;

- Submit the waiver request for the signature requirement in the 'Informed Consent' before participating in the minimal risk online survey that is 'a waiver of documentation of consent'.

- Submit the 'Informed Consent' (without any signature) in form of an introductory letter to decide whether agree or disagree to proceed in filling the provided online google form.

- Move the question to fill up the telephone number and the email address to the end of the google form and add the guarantee for strict confidentiality.

- Include the dissemination plan to the target audience, policy and program planners to promote immediate utilization of research results to strengthen the health systems 
Case study 2 focused the compliance with prevention guidelines and messages informing the general public about COVID-19 risks (Text box 2).

\section{Text box (2)}

Case study on compliance with prevention guidelines and messages informing the public about COVID-19 risks.

\section{Project summary}

It is a cross-sectional quantitative online survey among the general public using google forms to access the compliance with prevention guidelines for COVID-19 transmission. It aimed to find out the magnitude and predictors of public compliance with guidelines and information sources accessed by the public for prevention of COVID-19 transmission. The study population will be people 12 to 75 years old using Facebook and various social media and will collect the required data by requesting to complete the google survey form. The estimated sample size will be 171 .

In case study 2 , the IRB has identified the ethical concerns to

- Consider the inclusion of minors (12-17 years) in this online survey. However, it does not raise a big ethical concern due to not requiring privacy, not addressing the illegal issue or stigmatizing event and non-sensitive nature of questionnaire addressed in the google form. The questionnaires are mainly based upon government instructions widely available in the public media.

- Prepare and submit the 'Informed Consent'/'Assent' (without any signature) in form of an introductory letter to decide whether agree or disagree to proceed in filling the provided online google form.

- Submit the waiver request for the signature requirement in the 'Informed Consent'/'Assent' before participating in the minimal risk online survey that is a waiver of documentation of consent'.

- Include the dissemination plan of research results to the target audience, policy, and program planners to promote immediate utilization of research results to strengthen the healthcare infrastructure.

Case study 3 focused on epidemiological distribution and clinical characteristics of COVID-19 patients in country X (Text box 3). COVID-19 patients should be included in the vulnerable population especially when hospitalized and progressing into severity. However, this submitted proposal was categorized as a minimal risk study due to the use of secondary data extracted from case report forms (CRF).

\section{Text box (3)}

Case study on epidemiological distribution and clinical characteristics of Coronavirus disease (COVID-19).

\section{Project summary}

A cross-sectional quantitative study aimed to identify the epidemiological distribution, to describe the clinical and laboratory and radiological findings, to determine the proportion of symptomatic cases among COVID-19 cases, to evaluate the disease outcomes and to contribute the understanding of transmission of infection by assessing outcomes and management of the COVID-19 patients. It will analyse the publicly available secondary data of the Central Epidemiological Unit and case report forms recorded at the hospitals in Yangon Region which currently treat the COVID-19 confirmed cases.

The IRB further identified the ethical concerns in case study 3 to: 
- Consider the waiver of the informed consent as the data will be collected from the existing medical records covering the whole period of hospital stay;

- Observe anonymity while retrieving, analysing, and reporting the essential findings available from individual case report forms. However, it is critical to record the name of the patient in terms of numerical codes in a password protected computer and to allow the patient to request for corrections or suppression of the collected information before discharge.

Case study 4 focused safe and effective use of chemical disinfectants by general public and volunteers in non-healthcare settings of country X in the context of COVID-19 (Text box 4).

\section{Text box (4)}

Case study on safe and effective use of chemical disinfectants by general public and volunteers in non-healthcare settings in the context of COVID-19

\section{Project summary}

It is a cross-sectional mixed methods study to promote safe and effective use of chemical disinfectants among the users of online social media and volunteers in non-healthcare settings in the context of COVID-19. For quantitative data collection, general public and volunteers of civil society organization (CSO) will be recruited for self-reported structured questionnaires including knowledge, risk perception and practiced related to chemical disinfection and related health problems. For qualitative portion, telephone interviews will be done for 10 representatives of CSO about current practices, challenges of chemical disinfection in non-healthcare settings, and potential solutions for safe and appropriate use.

The IRB identified the ethical concerns in case study 4 to:

- Submit the 'Informed Consent' (without any signature) in form of an introductory letter to decide whether agree or disagree to proceed in filling the provided online google form;

- Submit the separate waiver request letter for the signature requirement in the 'Informed Consent' before participating in the minimal risk online survey that is 'a waiver of documentation of consent'

- Submit the request for verbal consent only for telephone interviews in collecting the qualitative data;

- Include in the consent for qualitative interview as: "It may need more than one call if any interruption or disturbance occurs meets during the call."

- Train data collectors for telephone interviews especially introduction, tone, and respect;

- Educate the volunteers on how to use the disinfectant properly after interviewing and refer to clinicians if they have any health problems related to disinfectant use.

Case study 5 focused on healthcare providers at the district level of healthcare infrastructure and their perceptions towards the feasibility of establishing community fever clinics by using mixed methods. (Text box 5).

The IRB identified the ethical concerns in case study 5 to:

- Consider the online data security via a video call on the Facebook messenger for qualitative interviews and to pay attention to the feeling of discomfort of being identified on Facebook;

- Maintain the confidentiality of collected data

- Prepare the 'waiver of documentation of informed consent'

- Disseminate through the virtual platform concerning the salient findings in form of aggregate data with concealment of personal identifiers 
Apparently, by participating in online surveys, none of the study participants could attain direct and immediate benefits. Nonetheless, the major benefit is the opportunity to contribute new knowledge. As for the incentives provided for participation in online surveys, four case studies in this research did not offer direct cash payment. However, interviewees through mobile phones and the Facebook messenger will be offered top-up phone bills to compensate their time.

\section{Text box (5)}

Case study on perception of health care providers on operational feasibility of community fever clinics

\section{Project summary}

A cross-sectional mixed methods study will identify the perception of both public and private health care providers on operational feasibility of community fever clinics in the district Y. For quantitative data collection, online Google forms will be used for 245 medical personnel. Altogether 14 key informants will be reached out for interviews via the video call using the face-book messenger.

\section{DISCUSSION}

The planned study design by the research team vis-a-vis their choices in the data collection portal (online system vs. conventional system) might have ethical implications for socio-behavioral and public health research [18-20] which is also true for research proposals related to COVID-19 infection [6-7]. In this research, all case studies illustrated the cross-sectional observational study design, and four out of five case studies involved digital platforms. Most of the researchers introduced the google forms via Facebook social media, and interviews through mobile phones. The internet research as a new avenue that has gained popularity in recent years is not without ethical challenges [21]. Among others, one case study preferred secondary data collection only by extracting the medical records to avoid the conduct of face-to-face interviews during the pandemic restrictions such as lock-down and semi-lock down measures and social distancing. Ethical concerns in record reviews highlight the importance of observing the anonymity [22]. It is emphasized as essential to keep the identifiers confidential in retrieving and reporting the findings in case study 3 through the extraction of available records of CRF at the study hospitals.

For digital data collection, the recruitment and eligibility criteria are important that requires an initial online announcement of the survey. The written informed consent procedures are not fit were not appropriate in this condition and an alternative form are was needed as evident in this brief review which is in consistency with other studies [23-25]. Therefore, as expressed in case studies, the IRB has suggested the researchers for the waiver request of the documented Informed Consent' in form of an introductory letter to decide whether agree or disagree to proceed in filling the online google forms. The IRB has to check the alternative consent taking procedures whether all the required information related to the study are informed provided to participants before data collection and proper archiving of consent like documentation in google data collection form and recording [26-28].

As reported by other studies, there was an increasing trend in using the social media platforms in the field of socio-behavioral research. This context may bring in complex ethical issues associated with using social media for data collection [29]. The IRB strongly recommended data collection training and pretesting for telephone interviews and other online media, to educate the participants about control measures of COVID-19 and to arrange for proper referral to those with reported physical or mental problems. The IRB needs to consider both ethical and technical issues affecting scientific integrity carefully in this situation by reviewing the data collection method and the inclusion of proper interviewer training. Data security was the priority for online research involving either active or passive data collection and also for mitigating the discomfort felt by participants being identified either in the social media platform or during the interviews with a 
video call from the Facebook messenger as noted in case study 5. Ethical issues were central to anonymity and confidentiality apart from data security. Within the European Union, studies need to comply with the General Data Protection Regulation (GDPR) in addition to country specific data protection regulations (In: EU Data Protection Rules and U.S. Implications, 2020) [30]. It is highly important to maintain the confidentiality of COVID-19 related data due to the possibility of stigmatization and violence. Therefore, the IRB needed to ensure thoroughly describing data storage and maintenance in the submitted proposals [30-32].

The dissemination of salient findings as an input to formulate new strategies for behavioral change communication, develop innovative public health interventions or to strengthen the existing policy and program guidelines are critical in public health and socio-behavioral research [32-33]. The IRB also suggested for the timely dissemination plan by virtual means during the pandemic to the target audience and the policy and program planners to promote immediate utilization. It is essential to check the involvement of dissemination plan about the study findings to relevant stakeholders, affected population and, the global community as necessary so that the greatest impact of the sociobehavioral and public health related research can be obtained. This fact has been supported by the COVID-19 Research Roadmap social science working group being established following the Global Research and Innovation meeting held in Geneva. Among others, one of the important responsibilities of the expert group members is to provide feedback on 'state of the art' COVID-19 evidence in priority areas [33-34]. As such, effective dissemination is critical to accelerate the timely access of stakeholders to evidence-based messages in locally relevant language supportive to mitigate the spread of corona virus.

\section{Conclusions}

A real-time evidence generated from this study could support the effective and efficient reviewing of research concerning public health emergency response by local IRBs, thereby promoting scientifically and ethically sound studies in similar scenarios. The IRBs need to handle the timely implementation of COVID-19 related studies by fulfilment of scientific integrity within a short processing time. In the long run, the enhancement in networking the IRBs in the Region could foster sharing of ways to deal with ethical dilemmas especially when using internet-based research and digital platforms in support of COVID-19 related socio-behavioral and public health research entity.

\section{REFERENCES}

1. Honey-Rosés J, Anguelovski I, Chireh VK, Daher C, Konijnendijk van den Bosch C, Litt JS, Mawani V, McCall MK, Orellana A, Oscilowicz E, Sánchez U. The impact of COVID-19 on public space: an early review of the emerging questions-design, perceptions and inequities. Cities \& Health 2020;8:1-7.

2. World Health Organisation. Ethical standards for research during public health emergencies: Distilling existing guidance to support COVID-19 R\&D. WHO/RFH/20.1.2020

3. Nuffield Council on Bioethics. Research in Global Health Emergencies [Internet], 2020. https://nuffieldbioethics.org/publications/research-in-global-health-emergencies.

4. Kantor BN, Kantor J. Non-pharmaceutical Interventions for Pandemic COVID-19: A CrossSectional Investigation of US General Public Beliefs, Attitudes, and Actions. Front Med 2020;7: 384.

5. Van Bavel JJ, Baicker K, Boggio PS, Capraro V, Cichocka A, Cikara M, Crockett MJ, Crum AJ, Douglas KM, Druckman JN, Drury J. Using social and behavioural science to support COVID-19 pandemic response. Nature Hum Behav 2020;4(5):460-71..

6. Qi,C, Zhu YC, Li CY, Hu YC, Liu LL, Zhang DD, Wang X, She KL., Jia, Y, Liu TX, Li XJ. Epidemiological characteristics and spatial-temporal analysis of COVID-19 in Shandong Province, China. Epidemiol Infection 2020;148:e141.

7. Wright KS. Ethical research in global health emergencies: making the case for a broader understanding of 'research ethics'. Int Health 2020;12(6):515-7.

8. Indian Council of Medical Research. National Guidelines for Ethics Committees reviewing Biomedical and Health Research during COVID-19 Pandemic. 2020. Available at: 
https://main.icmr.nic.in/sites/default/files/guidelines/EC_Guidance_COVID19_06_05_2020.p df

9. World Health Organization. Guidance for managing ethical issues in infectious disease outbreaks. World Health Organization. 2016. https://apps.who.int/iris/handle/10665/250580

10. Guidance for research ethics committees for rapid review of research during public health emergencies. World Health Organization. 2020.

11. Department of Health, Education, and Welfare; National Commission for the Protection of Human Subjects of Biomedical and Behavioral Research. The Belmont Report. Ethical principles and guidelines for the protection of human subjects of research. J Am Coll Dent 2014;81(3):4-13.

12. Saxena A, Horby P, Amuasi J, Aagaard N, Köhler J, Gooshki ES, Denis E, Reis AA, Ravinetto R. The ALERRT-WHO Workshop. Ethics Preparedness: Facilitating Ethics Review during Outbreaks - Recommendations from an Expert Panel. BMC Med Ethics 2019;20:29.

13. Kumar NK, Muthuswamy V. Fostering ethical biomedical and health research in India during the COVID-19 pandemic. Research Ethics 2020;16(3-4):1-10.

14. Mathur R. Ethics preparedness for infectious disease outbreaks research in India: A case for novel coronavirus disease 2019. Indian J Med Research 2020;151(2):124.

15. Wassie L, Gebre-Mariam S, Tarekegne G, Rennie S .Enhancing ethics review of social and behavioral research: developing a review template in Ethiopia. Research Ethics 2019;15(3-4):1-23.

16. MoHS. Updates on National COVID-19 Containment Strategies. In: Myanmar Health Sector Contingency Plan on COVID-19 and Other Emerging Respiratory Illnesses. 2020. http//:www.mohs.gov.mm

17. Pwint KH, Aung HM, Yadanar P, Aye NS, Show KL, et al. Expedient Reviews during the Covid19 Pandemic Period: Concept to Current Practice of the Institutional Review Board in the ResourceConstrained Scenario. J Bioethics Appl 2020;1(1).

18. Marshall PA. Ethical challenges in study design and informed consent for health research in resource-poor settings. (Special Topics in Social, Economic and Behavioural (SEB) Research report series; No. 5) (2007) TDR/SDR/SEB/ST/07.1

19. Zimmer M. But the data is already public: on the ethics of research in Facebook. Ethics Inf Technol. 2020.12(4):313-25.

20. Hunter RF, Gough A, O'Kane N, McKeown G, Fitzpatrick A, Walker T, McKinley M, Lee M, Kee F. Ethical Issues in Social Media Research for Public Health. Am J Pub Health 2018;108(3): 343-8.

21. Moreno MA, Goniu N, Moreno PS, Diekema D. Ethics of social media research: Common concerns and practical considerations. Cyberpsychol Behav Soc Network 2013;16(9):708-13.

22. International Ethical Guidelines for Biomedical Research Involving Human Subjects. Prepared by the Council for International Organizations of Medical Sciences (CIOMS) in collaboration with the World Health Organization (WHO) CIOMS Geneva 2002.

23. Whitaker C, Stevelink S, Fear N. The use of Facebook in recruiting participants for health research purposes: a systematic review. J Med Internet Res 2017;19(8):e290.

24. Russomanno, J, Patterson JG, Jabson Tree JM. Social Media Recruitment of Marginalized, Hardto-Reach Populations: Development of Recruitment and Monitoring Guidelines. JMIR Pub Health Surv 2019;5(4):e14886.

25. Osterrieder A, Poomchaichote T, Cuman G, et al.: Online survey questions: Social, ethical and behavioural aspects of COVID-19 (Version 1.024 April 2020). Zenodo. 2020. 10.5281 /zenodo. 3764913 .

26. Keller HE, Lee S. Ethical Issues Surrounding Human Participants Research Using the Internet, Ethics Behav 2003;13(3):211-9.

27. Buchanan EA, Hvizdak EE. Online survey tools: ethical and methodological concerns of human research ethics committees. J Empir Res Hum Res Ethics 2009;;4(2):37-48.

28. Roberts LD, Allen PJ. Exploring ethical issues associated with using online surveys in educational research, Educ Res Eval 2015;21(2):95-108.

29. Markham A., Buchanan E. Internet research: Ethical concerns. In Smelser, N. J., Baltes, P. B. (Eds.), International encyclopedia of social and behavioral science (pp. 606-613). Amsterdam, The Netherlands: Elsevier. 2015.

30. EU Data Protection Rules and U.S. Implications. Updated July 17 2020.Congressional Research Service.https://crs reports.congress.gov

31. Gupta S. Ethical issues in designing internet-based research: Recommendations for good practice. J Res Pract 2017;13(2).

32. Facca D, Smith MJ, Shelley J, Lizotte D, Donelle L. Exploring the ethical issues in research using digital data collection strategies with minors: A scoping review. PLoS One 2020;15(8):e0237875. 
41 Aye et al: Ethical Issues on Research Focused on the Containment of the COVID-19 Pandemic

33. McVay AB, Stamatakis KA, Jacobs JA, Tabak RG, Brownson RC. The role of researchers in disseminating evidence to public health practice settings: a cross-sectional study. Health Res Policy Syst 2016;14(1):1-9.

34. WHO R\&D Blueprint. Novel Coronavirus COVID-19 Social Science working group. World Health Organization, Geneva, 2020.

Acknowledgements: This paper has been presented at the $20^{\text {th }}$ International Online Conference of Forum for Ethical Review Committees in Asia and the western Pacific (FERCAP) (7-8 December 2020). Authors are indebted to Prof. Dr. Zaw Than Htun, Director General, Department of Medical Research for his encouragement and full support. Conflict of interest: Nil; Financial Disclosures: Nil 